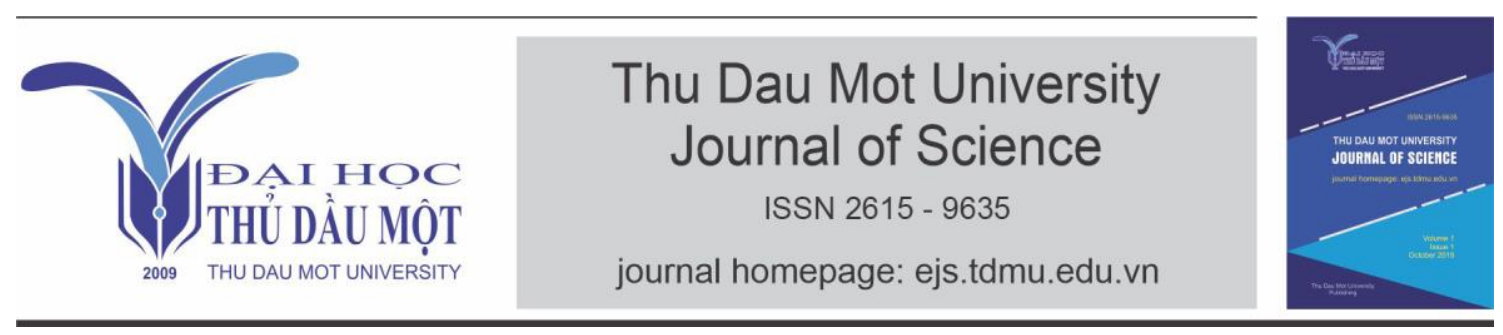

\title{
A study of fixed points and hopf bifurcation of hindmarsh- rose model
}

by Phan Van Long Em (An Giang university, Vietnam)

\author{
Article Info: $\quad$ Received 10 Sep. 2019, Accepted 20 Oct. 2019, Available online 15 Feb. 2020 \\ Corresponding author: pvlem@agu.edu.vn (Phan Van LongEm PhD) \\ https://doi.org/10.37550/tdmu.EJS/2020.01.002
}

\begin{abstract}
In this article, a class of Hindmarsh-Rose model is studied. First, all necessary conditions for the parameters of system are found in order to have one stable fixed point which presents the resting state for this famous model. After that, using the Hopf's theorem proofs analytically the existence of a Hopf bifurcation, which is a critical point where a system's stability switches and a periodic solution arises. More precisely, it is a local bifurcation in which a fixed point of a dynamical system loses stability, as a pair of complex conjugate eigenvalues cross the complex plane imaginary axis. Moreover, with the suitable assumptions for the dynamical system, a small-amplitude limit cycle branches from the fixed point.
\end{abstract}

Keywords: Hindmarsh-Rose model, fixed point, Hopf bifurcation, limit cycle

\section{Introduction}

In the beginning of 1980s, Hindmarsh J.L. and Rose R.M. studied a model called Hindmarsh-Rose model, to expose part of the inner working mechanism of the HodgkinHuxley equations, a famous model in study of neurophysiology since 1952. The Hindmarsh-Rose model was introduced as a dimensional reduction of the well-known Hodgkin-Huxley model (Hodgkin A. L., and Huxley A. F., 1952; Nagumo J., et al., 1962; 
Izhikevich E. M ., 2007; Ermentrout G. B., and Terman D. H ., 2009 ; Keener J. P., and Sney J., 2009 ; Murray J. D., 2010 ). It is constituted by two equations in two variables $u$ and $v$. The first one is the fast variable called excitatory representing the transmembrane voltage. The second variable is the slow recovery variable describing the time dependence of several physical quantities, such as the electrical conductance of the ion currents across the membrane. The Hindmarsh-Rose equations (HR) are given by

$$
\left\{\begin{array}{l}
\frac{d u}{d t}=u=f(u, v)=v-a u^{3}+b u^{2}+I, \\
\frac{d v}{d t}=v=g(u, v)=c-d u^{2}-v,
\end{array}\right.
$$

where $u$ corresponds to the membrane potential, $v$ corresponds to the slow flux ions through the membrane, $I$ corresponds to the applied extern current, and $a, b, c, d$ are parameters. Here, $I, a, b, c, d$ are real numbers.

The paper is organized as follows. In section 2, a study of fixed point is investigated and all necessary conditions for the parameters of Hindmarsh-Rose model are found in order to have a stable focus. In section 3, the system undergoes subcritical Hopf bifurcation is shown. And finally, conclusions are drawn in Section 4.

\section{A study of fixed points}

Equilibria or stability are tools to study the dynamic of fixed points. In mathematics, a fixed point of a function is an element of the function's domain that is mapped to itself by the function. This paper focuses on the fixed points of the system (1) given by the resolution of the following system

$$
\left\{\begin{array} { l } 
{ f ( u , v ) = 0 } \\
{ g ( u , v ) = 0 }
\end{array} \Leftrightarrow \left\{\begin{array}{l}
v-a u^{3}+b u^{2}+I=0 \\
v=c-d u^{2}
\end{array}\right.\right.
$$

It implies that

$$
a u^{3}+(d-b) u^{2}-c-I=0
$$

Let $\alpha=\frac{d-b}{a}$ and $\beta=-\frac{c+I}{a}$. The equation (2) can be written

$$
u^{3}+\alpha u^{2}+\beta=0
$$

To solve this equation, let's use the Cardan's formula after the following variables changes: 
Phan Van Long Em - Volume 2 - Issue 1-2020, p.98-109.

$$
u=\xi-\frac{d-b}{3 a}, p=-\frac{(d-b)^{2}}{3 a^{2}}, q=\frac{2(d-b)^{3}}{27 a^{3}}-\frac{c+I}{a}, \text { then } \xi^{3}+p \xi+q=0 .
$$

Let now $\Delta=4 p^{3}+27 q^{2}$.

If $\Delta>0$, then the equation (2) admits only one root and hence the system (1) admits a unique fixed point. Now, if $\Delta=0$, then the system (1) admits two fixed points, and finally if $\Delta<0$, the system (1) admits three fixed points (see Figure 1).

The Jacobian matrix of the system (1) is written as the following:

$$
A(u)=\left(\begin{array}{ll}
\frac{\partial f(u, v)}{\partial u} & \frac{\partial f(u, v)}{\partial v} \\
\frac{\partial g(u, v)}{\partial u} & \frac{\partial g(u, v)}{\partial v}
\end{array}\right)=\left(\begin{array}{cc}
-3 a u^{2}+2 b u & 1 \\
-2 d u & -1
\end{array}\right)
$$

Let $\left(u^{*}, v^{*}\right)$ be one fixed point of (1), we have

$\operatorname{Det}\left(A\left(u^{*}\right)-\lambda \boldsymbol{I}_{2}\right)=\lambda^{2}-\operatorname{Tr}\left(A\left(u^{*}\right)\right) \lambda+\operatorname{Det}\left(A\left(u^{*}\right)\right)$,

where $\operatorname{Tr}(A(u))=-3 a u^{2}+6 u-1$ and $\operatorname{Det}(A(u))=3 a u^{2}+4 u$.

The reduced discriminant of $\operatorname{Tr}(A(u))$ is $\Delta^{\prime}=b^{2}-3 a$. If $b^{2}>3 a$, then $\operatorname{Tr}(A(u))$ admits two real roots given by

$u_{T r 1}=\frac{b-\sqrt{b^{2}-3 a}}{3 a}=\frac{b-D}{3 a}$ and $u_{T r 2}=\frac{b+\sqrt{b^{2}-3 a}}{3 a}=\frac{b+D}{3 a}$ with $D=\sqrt{b^{2}-3 a}$.

Two roots of $\operatorname{Det}(A(u))$ is

$u_{\text {Det } 1}=\frac{(d-b)-\sqrt{(d-b)^{2}}}{-3 a}=-2 \frac{d-b}{3 a}$ and $u_{\text {Det } 2}=\frac{(d-b)+\sqrt{(d-b)^{2}}}{-3 a}=0$.

The nature of fixed points is rapported in Table 1.

TABLE 1: Stability of fixed point

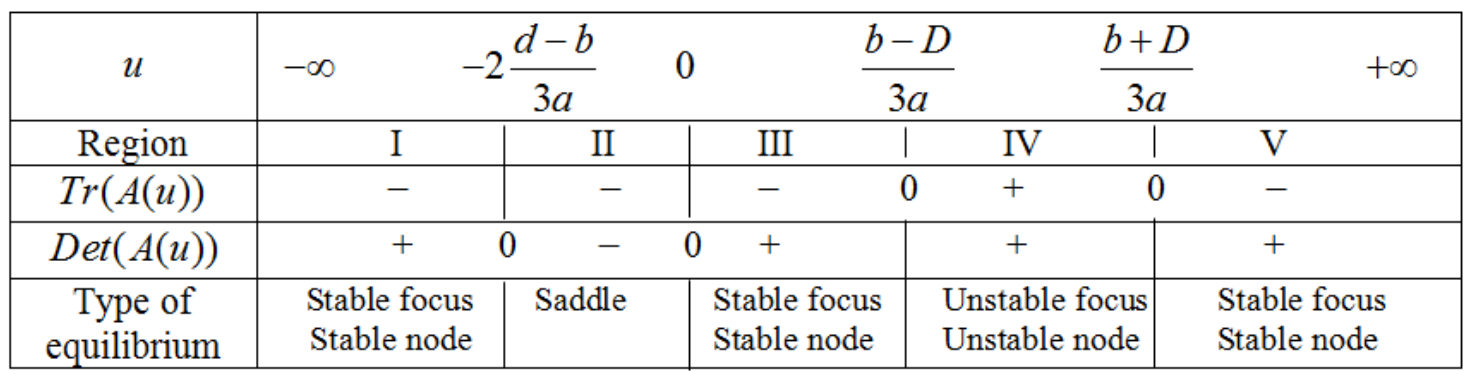

If $b^{2}<3 a$, then $\operatorname{Tr}(A(u))<0$ for all values of $u$ and in this case, the fixed point is only stable focus or stable node. Morever, in this study, the model is needed to generate the 
potential actions, it is necessary for the existence of a limit cycle. In the other word, it is need to have an unstable focus or a center. So the condition $b^{2} \geq 3 a$ is chosen to be in the region IV of Table 1. The infimum and superimum in the region IV are given by $L=\frac{b-D}{3 a}$ and $M=\frac{b+D}{3 a}$.

To observe the behavior of the system (1) like Figure 1, we fix the values of parameters as the following $a=1, b=3, c=1, d=5, I=0$. Then, the system (1) becomes

$$
\left\{\begin{array}{l}
\frac{d u}{d t}=v-u^{3}+3 u^{2} \\
\frac{d v}{d t}=1-5 u^{2}-v
\end{array}\right.
$$

The system (3) has three fixed points:

$A=(-1.618033989,-12.090169948), B=(-1,-4), C=(0.618033989,-0.909830058)$.

In Figure 1(a), we simulated two nullclines, $u=0$ in red and $\stackrel{\square}{\square}=0$ in green. The intersection point of these two nullclines is three fixed points $A, B, C$ and one orbit of (3) is represented in blue and it is a limit cycle.

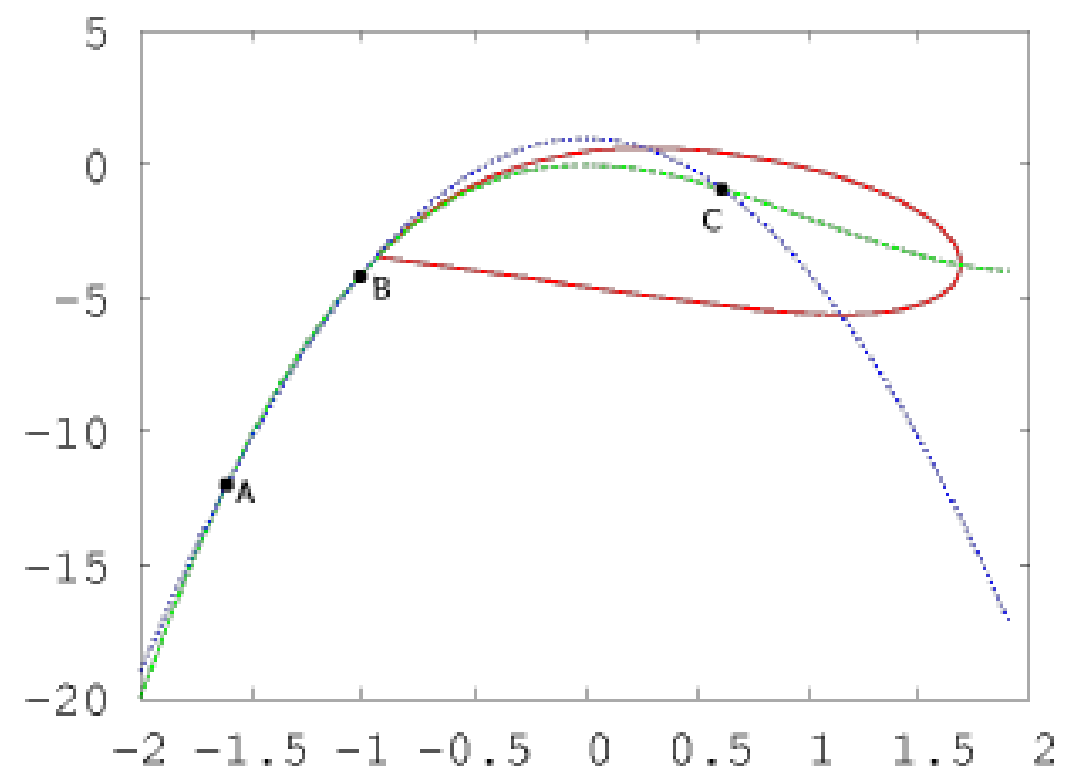

Figure 1: Numerical results obtained for two nullclines $u=0$ in green and $v=0$ in blue. The intersection points are fixed points A, B and C. The red curve is the limit cycle.

At the point $A$, we get $\operatorname{Det}(A)=1.381966013$ and $\operatorname{Tr}(A)=-18.562305903$, so $A$ is a stable node. At the point $B$, we get $\operatorname{Det}(B)=-1$ and $\operatorname{Tr}(B)=-10$, hence $B$ is a 
Phan Van Long Em - Volume 2 - Issue 1-2020, p.98-109.

saddle. At the point $C$, we get $\operatorname{Det}(C)=3.618033991$ and $\operatorname{Tr}(C)=1.562305899$, so $C$ is a instable focus.

\section{3. existence and direction of hopf bifurcation}

This section focuses on the existence and the direction of Hopf bifurcation, which corresponds to the passage of a fixed point to a limit cycle under the effect of variation of a parameter. Recall the Hopf's theorem (Dang-Vu Huyen, and Delcarte C., 2000).

Theorem 1. Consider the system of two ordinary differential equations

$$
\left\{\begin{array}{l}
u=f(u, v, a) \\
v=g(u, v, a)
\end{array}\right.
$$

Let $\left(u^{*}, v^{*}\right)$ a fixed point of the system (4) for all $a$. If the Jacobian matrix of the system (4) at $\left(u^{*}, v^{*}\right)$ admits two conjugate complex eigenvalues, $\lambda_{1,2}(a)=\alpha(a) \pm i w(a)$ and there is a certain value $a=a_{c}$ such that

$$
\alpha\left(a_{c}\right)=0, w\left(a_{c}\right) \neq 0 \text { and } \frac{\partial \alpha(a)}{\partial a}\left(a_{c}\right) \neq 0 .
$$

Then, a Hopf bifurcation survives when the value of bifurcation parameter a passes by $a_{c}$ and $\left(u^{*}, v^{*}, a_{c}\right)$ is a point of Hopf bifurcation. Moreover, let $c_{1}$ in order that

$$
\begin{aligned}
c_{1} & =\frac{1}{16 w\left(a_{c}\right)}\left(-\frac{\partial^{2} F}{\partial u^{2}} \frac{\partial^{2} G}{\partial u^{2}}+\frac{\partial^{2} F}{\partial u^{2}} \frac{\partial^{2} F}{\partial u \partial v}-\frac{\partial^{2} G}{\partial u^{2}} \frac{\partial^{2} G}{\partial u \partial v}\right. \\
- & \left.\frac{\partial^{2} G}{\partial v^{2}} \frac{\partial^{2} G}{\partial u \partial v}+\frac{\partial^{2} F}{\partial v^{2}} \frac{\partial^{2} F}{\partial u \partial v}+\frac{\partial^{2} F}{\partial v^{2}} \frac{\partial^{2} G}{\partial v^{2}}\right)+\left(\frac{\partial^{3} F}{\partial u^{3}}+\frac{\partial^{3} F}{\partial u \partial v^{2}} \frac{\partial^{3} G}{\partial u^{2} \partial v}+\frac{\partial^{3} G}{\partial v^{3}}\right),
\end{aligned}
$$

where $F$ and $G$ are given by the method of Hassard, Kazarinoff and Wan (Dang-Vu Huyen, and Delcarte C., 2000).

We can distinguish different cases

TABLE 2: Stability of the fixed points according to Hopf bifurcation

\begin{tabular}{|l|l|l|l|}
\hline & & $c_{1}<0$ & $c_{1}>0$ \\
\hline \multirow{2}{*}{$\frac{\partial \alpha}{\partial a}\left(a_{c}\right)>0$} & $a<a_{c}$ & $\begin{array}{l}\text { stable equilibrium } \\
\text { and no periodic orbit }\end{array}$ & $\begin{array}{l}\text { stable equilibrium } \\
\text { and unstable periodic orbit }\end{array}$ \\
\cline { 2 - 4 } & $a>a_{c}$ & $\begin{array}{l}\text { unstable equilibrium } \\
\text { and stable periodic orbit }\end{array}$ & $\begin{array}{l}\text { unstable equilibrium } \\
\text { and no periodic orbit }\end{array}$ \\
\hline
\end{tabular}


Thu Dau Mot University Journal of Science - Volume 2 - Issue 1-2020

\begin{tabular}{|l|l|l|l|}
\hline \multirow{2}{*}{$\frac{\partial \alpha}{\partial a}\left(a_{c}\right)<0$} & $a<a_{c}$ & $\begin{array}{l}\text { unstable equilibrium } \\
\text { and stable periodic orbit }\end{array}$ & $\begin{array}{l}\text { unstable equilibrium } \\
\text { and periodic orbit }\end{array}$ \\
\cline { 2 - 4 } & $a>a_{c}$ & $\begin{array}{l}\text { stable equilibrium } \\
\text { and no periodic orbit }\end{array}$ & $\begin{array}{l}\text { stable equilibrium } \\
\text { and unstable periodic orbit }\end{array}$ \\
\hline
\end{tabular}

Now this theorem is applied to the Hindmarsh-Rose model in which $a$ represents the bifurcation parameter

$$
\left\{\begin{array}{l}
\frac{d u}{d t}=v-a u^{3}+3 u^{2}+I \\
\frac{d v}{d t}=1-5 u^{2}-v
\end{array}\right.
$$

Let $\left(u^{*}, v^{*}\right)$ a fixed point of the system (6). Let $u=u_{1}+u^{*}$ and $v=v_{1}+v^{*}$, then

$$
\left\{\begin{array}{l}
u_{1}=f\left(u_{1}, v_{1}, a\right)=\left(v_{1}+v^{*}\right)-a\left(u_{1}+u^{*}\right)^{3}+3\left(u_{1}+u^{*}\right)^{2}+I \\
v_{1}=g\left(u_{1}, v_{1}, a\right)=1-5\left(u_{1}+u^{*}\right)^{2}-\left(v_{1}+v^{*}\right)
\end{array}\right.
$$

With a development of the functions $f$ and $g$ at the neighborhood of $(0,0, a)$, the above systems become

$$
\left\{\begin{array}{l}
u_{1}=u_{1} \frac{\partial f}{\partial u_{1}}(0,0, a)+v_{1} \frac{\partial f}{\partial v_{1}}(0,0, a)+F\left(u_{1}, v_{1}, a\right) \\
v_{1}=u_{1} \frac{\partial g}{\partial u_{1}}(0,0, a)+v_{1} \frac{\partial g}{\partial v_{1}}(0,0, a)+G\left(u_{1}, v_{1}, a\right)
\end{array}\right.
$$

where $F\left(u_{1}, v_{1}, a\right)$ and $G\left(u_{1}, v_{1}, a\right)$ are the nonlinear terms, then

$$
\left\{\begin{array}{l}
u_{1}=\left(-3 a u^{2}+6 u^{*}\right) u_{1}+v_{1}+F\left(u_{1}, v_{1}, a\right) \\
v_{1}=-10 u * u_{1}-v_{1}+G\left(u_{1}, v_{1}, a\right)
\end{array}\right.
$$

with $F\left(u_{1}, v_{1}, a\right)=-a u_{1}^{3}+\left(-3 a u^{*}+3\right) u_{1}^{2}$ and $G\left(u_{1}, v_{1}, a\right)=-5 u_{1}^{2}$.

Now, $(0,0, a)$ is a fixed point of the system. The Jacobian matrix is given by

$$
A=\left(\begin{array}{cc}
-3 a u^{* 2}+6 u * & 1 \\
-10 u * & -1
\end{array}\right)
$$


Phan Van Long Em - Volume 2 - Issue 1-2020, p.98-109.

The characteristic polynomial

$\operatorname{Det}\left(A-\lambda \boldsymbol{I}_{2}\right)=\lambda^{2}+\left(3 a u^{* 2}-6 u *+1\right) \lambda+3 a u^{* 2}+4 u *$.

Let $P(a)=-\operatorname{Tr}(A)$ and $Q(a)=\operatorname{Det}(A)$. We get

$\lambda^{2}+P(a) \lambda+Q(a)=0$

Hence, the Jacobian matrix admits a pair of conjugate complex eigenvalues if $\operatorname{Det}(A)>\frac{1}{4} \operatorname{Tr}(A)^{2}$ and the above equation has the following roots

$\lambda_{1,2}=\alpha(a) \pm i w(a)$

with $\alpha(a)=-\frac{3 a *^{2}-6 u^{*}+1}{2}$ and $w(a)=\sqrt{3 a u^{2}+4 u *-\alpha(a)^{2}}$.

Moreover, the value $a_{c}$ of $a$, for which the real part of these eigenvalues is null, is given by the equations $P\left(a_{c}\right)=0$ and $Q\left(a_{c}\right)>0$, then

$a_{c}=\frac{6 u^{*}-1}{3 u^{*}}$ and $a_{c}>\frac{-4}{3 u^{*}} \Rightarrow u^{*}>\frac{1}{10}$.

Moreover, $\frac{\partial \alpha}{\partial a}\left(a_{c}\right)=-\frac{3 u^{* 2}}{2}$.

Thus, $\alpha\left(a_{c}\right)=0, w\left(a_{c}\right) \neq 0$ and $\frac{\partial \alpha(I)}{\partial a}\left(a_{c}\right) \neq 0$, then $a_{c}$ is a bifurcation Hopf value of the parameter $a$.

In the following, the direction and the stability of Hopf bifurcation are investigated. To do this, let's determine an eigenvector $v_{1}$ associated with the eigenvalue $\lambda_{1}$, obtained by resolving the system

$$
\left(A-\lambda_{1} \boldsymbol{I}_{2}\right)\left(\begin{array}{l}
u \\
v
\end{array}\right)=0 \Rightarrow\left\{\begin{array}{l}
\left(1+i \sqrt{10 u^{*}-1}\right) u+v=0 \\
-10 u * u+\left(-1+i \sqrt{10 u^{*}-1}\right) v=0
\end{array}\right.
$$

A solution of this system is an eigenvector associated with $\lambda_{1}$ given by

$$
V_{1}=\left(\begin{array}{c}
1 \\
-1-i \sqrt{10 u^{*}-1}
\end{array}\right)
$$

The base change matrix is given by 


$$
P=\left(\operatorname{Re}\left(V_{1}\right)-\operatorname{Im}\left(V_{1}\right)\right)=\left(\begin{array}{cc}
1 & 0 \\
-1 & \sqrt{10 u^{*}-1}
\end{array}\right)
$$

Then

$$
P^{-1}=\frac{1}{\sqrt{10 u^{*}-1}}\left(\begin{array}{cc}
\sqrt{10 u^{*}-1} & 0 \\
1 & 1
\end{array}\right)
$$

Now let the variable change

$$
\left(\begin{array}{l}
u_{1} \\
v_{1}
\end{array}\right)=P\left(\begin{array}{l}
u_{2} \\
v_{2}
\end{array}\right) \Rightarrow\left(\begin{array}{l}
u_{2} \\
v_{2}
\end{array}\right)=P^{-1}\left(\begin{array}{l}
u_{1} \\
v_{1}
\end{array}\right)
$$

Hence

$$
\left(\begin{array}{c}
\square \\
u_{2} \\
\square \\
v_{2}
\end{array}\right)=P^{-1}\left(\begin{array}{c}
\square \\
u_{1} \\
\square \\
v_{1}
\end{array}\right)=P^{-1} A P\left(\begin{array}{l}
u_{2} \\
v_{2}
\end{array}\right)+P^{-1}\left(\begin{array}{c}
F\left(u_{2}, v_{2}, a\right) \\
G\left(u_{2}, v_{2}, a\right)
\end{array}\right) .
$$

Let $A^{\prime}(a)=P^{-1} A P=\left(\begin{array}{cc}\alpha(a) & -w(a) \\ w(a) & \alpha(a)\end{array}\right)$. Then, for $a=a_{c}$, it implies that

$$
A^{\prime}\left(a_{c}\right)=\left(\begin{array}{cc}
0 & -w\left(a_{c}\right) \\
w\left(a_{c}\right) & 0
\end{array}\right) \Rightarrow\left\{\begin{array}{l}
\square=-w\left(a_{c}\right) v_{2}+F\left(u_{2}, v_{2}, a_{c}\right) \\
v_{2}=w\left(a_{c}\right) u_{2}+G\left(u_{2}, v_{2}, a_{c}\right)
\end{array}\right.
$$

with

$$
\left(\begin{array}{l}
F\left(u_{2}, v_{2}, a_{c}\right) \\
G\left(u_{2}, v_{2}, a_{c}\right)
\end{array}\right)=P^{-1}\left(\begin{array}{l}
F\left(u_{2}, v_{2}, a_{c}\right) \\
G\left(u_{2}, v_{2}, a_{c}\right)
\end{array}\right)
$$

Then

$$
\left\{\begin{array}{l}
F\left(u_{2}, v_{2}, a\right)=-a u_{2}^{3}+\left(-3 a u^{*}+3\right) u_{2}^{2} \\
G\left(u_{2}, v_{2}, a\right)=\frac{1}{\sqrt{10 u^{*}-1}}\left(-a u_{2}^{3}-\left(3 a u^{*}+2\right) u_{2}^{2}\right)
\end{array}\right.
$$

Let $c_{1}$ be given by the equation (5). The functions $F$ and $G$ depend only on $u_{2}$, the coefficient $c_{1}$ is given by

$$
c_{1}=-\frac{1}{16 w\left(a_{c}\right)} \frac{\partial^{2} F}{\partial u_{2}^{2}}\left(0,0, a_{c}\right) \frac{\partial^{2} G}{\partial u_{2}^{2}}\left(0,0, a_{c}\right)+\frac{\partial^{3} F}{\partial u_{2}^{3}}\left(0,0, a_{c}\right)
$$


Phan Van Long Em - Volume 2 - Issue 1-2020, p.98-109.

At the point $\left(u_{2}, v_{2}\right)=(0,0)$ and for $a=a_{c}$, it implies that $w\left(a_{c}\right)=\sqrt{10 u^{*}-1}$, and

$$
\begin{aligned}
c_{1} & =-6 a_{c}+\frac{1}{16 \sqrt{10 u^{*}-1}} \cdot \frac{4}{\sqrt{10 u^{*}-1}}\left(-3 a_{c} u^{*+3}\right)\left(3 a_{c} u^{*+2}\right) \\
& =-6 a_{c}+\frac{3}{4\left(10 u^{*}-1\right)}\left(-3 a_{c}^{2} u^{* 2}+a_{c} u^{*+2}\right) .
\end{aligned}
$$

Theorem 1 permits to deduce the direction and the stability of Hopf bifurcation from the signs of $\frac{\partial \alpha}{\partial a}\left(a_{c}\right)$ and $c_{1}$. Now we apply this theorem in fixing all parameters values except the bifurcation parmaeter $a$. Let $I=0$, the system (6) becomes

$$
\left\{\begin{array}{l}
\frac{d u}{d t}=v-a u^{3}+3 u^{2} \\
\frac{d v}{d t}=1-5 u^{2}-v
\end{array}\right.
$$

The fixed points are given by resolving the equation $u^{3}+\frac{2}{a} u^{2}-\frac{1}{a}=0$.

Let

$$
u=\xi-\frac{2}{3 a}, p=-\frac{4^{2}}{3 a^{2}}, q=\frac{16}{27 a^{3}}-\frac{1}{a},
$$

then $\xi^{3}+p \xi+q=0$. Let now $\Delta=4 p^{3}+27 q^{2}$. We choose arbitrarily one condition over $a$, in order to have only a fixed point, it means

$$
\Delta>0 \Rightarrow a \in\left(-\infty ;-\frac{4 \sqrt{2}}{3 \sqrt{3}}\right) \cup\left(\frac{4 \sqrt{2}}{3 \sqrt{3}} ;+\infty\right)
$$

With those values of $a$, we get

$$
\begin{aligned}
u *(a)= & \frac{\left(9 a \sqrt{27 a^{2}-32}+27 \sqrt{3} a^{2}-16 \sqrt{3}\right)^{\frac{2}{3}}}{32^{\frac{1}{3}} 3^{\frac{1}{6}} a\left(9 a \sqrt{27 a^{2}-32}+27 \sqrt{3} a^{2}-16 \sqrt{3}\right)^{\frac{1}{3}}} \\
& -\frac{22^{\frac{1}{3}} 3^{\frac{1}{6}}\left(9 a \sqrt{27 a^{2}-32}+27 \sqrt{3} a^{2}-16 \sqrt{3}\right)^{\frac{1}{3}}-42^{\frac{2}{3}} 3^{\frac{1}{3}}}{3.2^{\frac{1}{3}} 3^{\frac{1}{6}} a\left(9 a \sqrt{27 a^{2}-32}+27 \sqrt{3} a^{2}-16 \sqrt{3}\right)^{\frac{1}{3}}} .
\end{aligned}
$$


Then, $a_{c}=\frac{6 u *(a)-1}{3 u *(a)^{2}}$. Moreover, $a_{c}$ is solution of the equation

$$
\frac{6 u *(a)-1}{3 u *(a)^{2}}-a=0 .
$$

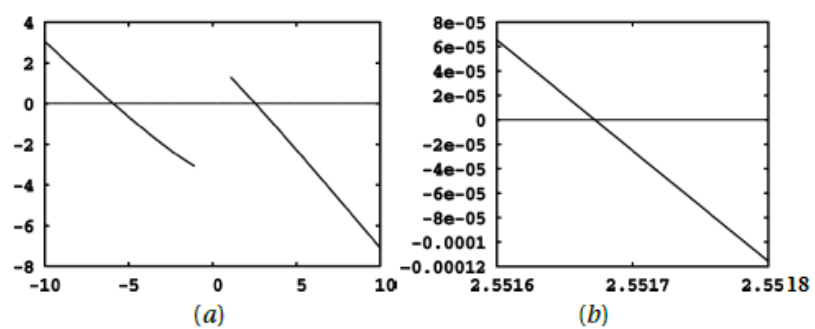

Figure 2: (a) The resolution of the equation (8) gives two solutions over $[-10 ; 10]$, corresponding to the intersections with the abscisses axis. (b) We are interested in the case where $a>0$, so $a_{c} \in[2.55165 ; 2.5517]$

The graphic resolution of the equation (8) gives two solutions over [-10;10] (see Figure 2(a)). Here, we are interested in the case where $a>0$, so $a_{c} \in[2.55165 ; 2.5517]$ (see Figure 2(b)). With these values of $a_{c}$, we get

$$
u^{*} \approx 0.54>\frac{1}{10}, 3 a_{c} u^{*^{2}}+4 u^{*} \approx 4.392187794>\frac{1}{4}\left(-3 a_{c} u^{*^{2}+6 u *-1}\right)^{2} \approx 1.526 .10^{-5}
$$

Moreover, $c_{1}=-6 a_{c}+\frac{3}{4(10 u *-1)}\left(-3 a_{c}^{2} u^{* 2}+a_{c} u^{*}+2\right) \approx-15.632152<0$.

So, we have $\quad c_{1}<0, \frac{\partial \alpha}{\partial a}\left(a_{c}\right)<0 . \quad$ From $\quad$ Theorem 1, $\left(u^{*}, v^{*}, a_{c}\right)=\left(0.54,-0.46, a_{c} \approx 2.551655\right)$ is a Hopf bifurcation point. Moreover, for $a<a_{c}$, the fixed point is unstable with a stable periodic orbit; while for $a>a_{c}$, the fixed point is stable without periodic orbit (see Figure 3). Figure 3(a) shows the phase portrait in the plane $(u, v)$ of the system (7) with $a=2.54$, and a stable limit cycle for a value $a=2.54<a_{c}$. Figure 3(b) presents the time series corresponding to $(t, u)$. Figure 3(c) shows the phase portrait in the plane $(u, v)$ of the system (7) with $a=2.57$, and a focus stable for a value $a=2.57>I_{c}$. Figure $3(\mathrm{~d})$ presents the time series corresponding to $(t, u)$. 
Phan Van Long Em - Volume 2 - Issue 1-2020, p.98-109.
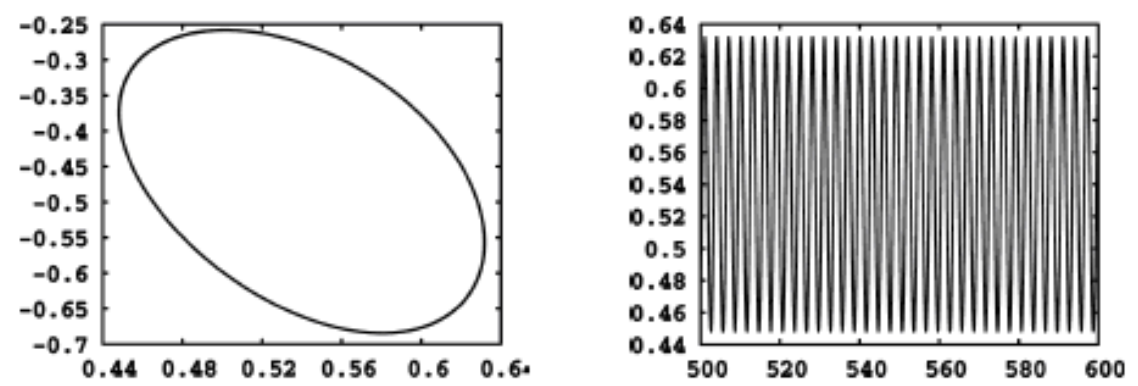

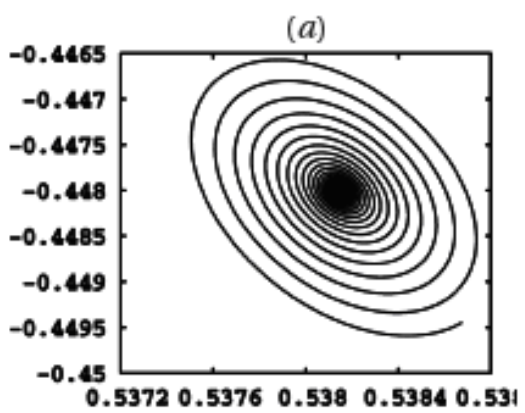

(c)

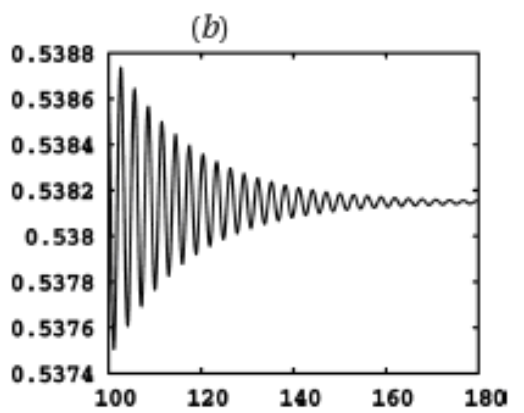

(d)

Figure 3: (a) Phase portrait in the plane $(u, v)$ of the system (7) with $a=2.54$, and a stable limit cycle for a value $a=2.54<a_{c}$. (b) Time series corresponding to $(t, u)$. (c) Phase portrait in the plane $(u, v)$ of the system (7) with $a=2.57$, and a focus stable for a value $a=2.57>I_{c}$. (d) Time series corresponding to $(t, u)$

\section{Conclusion}

This work showed the necessary conditions for the parameters of Hindmarsh-Rose model such that there exists only a stable fixed point. It represents the resting state in this system. The parameter $a$ is chosen like a bifurcation parameter, and when it crosses through the bifurcations values, then the equilibrium point loses its stability and becomes a limit cycle that implies the existence of a Hopf bifurcation. In this paper, the Hindmarsh-Rose model has one bifurcation value where there exists the subcritical Hopf bifurcation. The future work will be studied about the chaos properties in the Hindmarsh-Rose by adding some perturbation parameters.

\section{References}

Arena P., Fortuna L., Frasca M., La RosaM., (2006). Locally active Hindmarsh-Rose neurons, Chaos Sol. and Fract. 27:405-412.

Dang-Vu Huyen, and Delcarte, C., (2000). Bifurcations and Chaos, an introduction to dynamicscontemporary with programs in Pascal, Fortan et Mathematica. Eds Elipses, Université - Mécanique (in french). 
Ermentrout, G. B., Terman, D. H., (2009). Mathematical Foundations of Neurosciences. Springer.

Hodgkin, A.L., and Huxley, A. F., (1952). A quantitative description of membrane current and its application to conduction and excitation in nerve. J. Physiol. 117: 500-544.

Izhikevich, E. M., (2007). Dynamical Systems in Neuroscience. The MIT Press.

Keener, J. P., and Sneyd, J., (2009). Mathematical Physiology. Springer.

Murray, J. D., (2010). Mathematical Biology. Springer.

Nagumo, J., Arimoto, S., and Yoshizawa, S., (1962). An active pulse transmission line simulating nerve axon. Proc. IRE. 50: 2061-2070.

Nikolov S., (2005). An alternative bifurcation analysis of the Rose-Hindmarsh model, Chaos Solitons and Fractal. 23:1643-1649. 